\title{
LA DIMENSIÓN NOÉTICA DE LA SALUD EN LA LOGOTERAPIA DE VIKTOR FRANKL
}

\section{THE NOETIC DIMENSION OF HEALTH IN VIKTOR FRANKL'S LOGOTHERAPY}

\section{Raquel Ferrández Formoso (UNED)}

Recibido 2 mayo 2019

Aceptado 22 septiembre 2019

Resumen: En este artículo abordamos los conceptos de "salud» y «enfermedad» tal y como han sido entendidos en el seno de la Logoterapia del psiquiatra vienés Viktor Frankl, uno de los métodos psicoterapéuticos más importantes del siglo XX. Esta psicoterapia existencial otorga preeminencia a la dimensión noética o espiritual de la salud, que ha sido, sin embargo, totalmente obviada en el debate naturalismo/normativismo propio de la filosofía de la medicina. Por ello, en este escrito tratamos de mostrar de qué modo las aportaciones teóricas de Viktor Frankl pueden contribuir a la discusión filosófica acerca del estatuto ontológico de la enfermedad, especialmente en el ámbito polémico de la psiquiatría.

Palabras clave: salud; naturalismo; normativismo; psiquiatría humanista; biopsiquiatría;

\begin{abstract}
The aim of this paper is to examine the concepts of "health" and "disease" as they have been understood by the Logotherapy of the Viennese psychiatrist Viktor Frankl, one of the most important psychotherapeutic methods of the twentieth century. This existential psychotherapy gives preeminence to the noetic or spiritual dimension of health, which has been totally ignored in the naturalism/normativism debate proper of the Philosophy of Medicine. Therefore, our main objective is to show how the theoretical approach of Viktor Frankl can contribute to the philosophical discussion about the ontological status of the disease, especially in the controversial field of psychiatry.
\end{abstract}

Key words: health; naturalism;normativism; humanistic psychiatry, biopsychiatry; 


\section{Introducción}

La logoterapia es un método psicoterapéutico creado por el psiquiatra austríaco Viktor Frankl (1905-1997), y englobado en la corriente denominada "psiquiatría existencial». En el ámbito de la psicología forma parte del movimiento humanístico de la "tercera fuerza», característico de los años 1960, -década en la que la psicología humanista fue aceptada por la American Psychological Association (APA)-, que surge para hacer frente a las otras «dos fuerzas» dominantes, a saber: el psicoanálisis de Sigmund Freud y la psicología individual de Alfred Adler ${ }^{1}$. La logoterapia parte, en primer lugar, de una antropología humanista, se apoya, seguidamente, en valores existencialistas (tales como la voluntad, la libertad y la responsabilidad) y, finalmente, lleva a la práctica clínica el método fenomenológico basado en la epojé, el autodistanciamiento y la autotrascendencia. Considerada por algunos autores como la tercera escuela de psicoterapia vienesa (Tobías y García, 2009:150) constituye la aplicación del existenzanalyse o «análisis existencial» de Viktor Frankl, término que su autor no emplea demasiado con el fin de evitar confusiones con otra psicoterapia contemporánea, el daseinanalyze, del psiquiatra suizo L. Binswanger, también incluido en el marco de la psiquiatría fenomenológico-existencial.

En este escrito abordaremos los rasgos específicos que distinguen a la logoterapia de otras vertientes terapéuticas existenciales, pero, sobre todo, haremos hincapié en lo que diferencia a la psiquiatría existencial frente al paradigma dominante en la actualidad, la denominada «biopsiquiatría» o psiquiatría biológica. Mientras la primera da prioridad al tratamiento psicoterapéutico en el caso de trastornos para los que no existe un sustrato biológico evidente, la segunda entiende que todas las enfermedades mentales poseen tal sustrato, y se caracteriza por su relación estrecha con la farmacoterapia. Esta distinción a nivel metodológico abre el debate sobre si ambas terapéuticas son excluyentes o si, por el contrario, pueden aplicarse al paciente como componentes de un tratamiento integral. La biopsiquiatría, se apoya a nivel teórico en la neurobiología y en la fisiología en general, así como en un enfoque empirista del que carecen, precisamente, los postulados fenomenológicos y existencialistas en los que se basa la psiquiatría existencial; por otro lado, la concepción naturalista del ser humano acerca la biopsiquiatría al mecanicismo y lo enfrenta al humanismo, perspectiva que caracteriza a la psiquiatría existencial. Al mismo tiempo, el empleo frecuente de la farmacoterapia relaciona el paradigma biologicista con lo que el psiquiatra norteamericano Frances Allen denomina la «inflación del diagnóstico» en el ámbito psiquiátrico,

1. Para más información sobre la historia y los orígenes de «la tercera fuerza de la psicología», Cf. Brennan, J.:Historia y sistemas de la psicología. México: Practice hall, 1999, pp. 288-306.

Thémata. Revista de Filosofía No60 (2019) pp.: 35-48. 
dado el fuerte incremento de «enfermedades» recogidas en la quinta edición del Manual diagnóstico y estadístico de los trastornos mentales (DSM-5; publicado en 2013) con respecto a las ediciones anteriores. Con todo, se hace necesario tratar con anterioridad otra cuestión de carácter ontológico que afecta a todos los ámbitos de la medicina y que ha acaparado durante décadas un debate interminable: la naturaleza misma de la «enfermedad» y la «salud». Si las propuestas varían en el dominio de la medicina somática, en el terreno psiquiátrico esta cuestión se vuelve todavía más ambigua. Sin llegar a resolver si la enfermedad y la salud son hechos objetivos y libres de valores (naturalismo) o si son meros valores normativos o construcciones sociales (normativismo), no pocos autores y psiquiatras defienden que la mayor parte de las enfermedades mentales presentes en el DSM-5 (y en ediciones anteriores) no son descubiertas sino «inventadas». Tal y como expone Ethan Watters (2010:291), durante el período de elaboración del DSM-5, en la página web de la APA el público tenía habilitadas dos opciones de sugerencia: podía proponer «la eliminación de un desorden existente», o bien, sugerir "un nuevo desorden» con el fin de que fuese valorado para su incorporación al DSM-5. Esta iniciativa democrática parece menos frecuente en el ámbito de la medicina somática. Para definir la enfermedad en el ámbito psiquiátrico, en muchas ocasiones se ha tenido en cuenta la opinión pública, y el contexto socio-político, como lo prueba el hecho de que la homosexualidad se incluyese como trastorno mental en el DSM-1, publicado en 1952, pero ya no en el DSM-2 aparecido en un año revolucionario, 1968. (Aucouturier y Demazeux, 2012:84-85). De ahí que nos preguntemos, a lo largo de este escrito, por el lugar donde cabría situar a la logoterapia en el seno de este debate, teniendo en cuenta que, como Viktor Frankl (2008: 26) la describe, se trata una "psicoterapia que "arranca de lo espiritual"» y propone, de este modo, el carácter noógeno (espiritual, noético) de muchos de los estados sufrientes considerados por la ciencia médica como «neurosis psicológicas».

\section{Logoterapia: la dimensión noética de la salud}

En el ámbito de la psiquiatría, el estatuto ontológico de la enfermedad varía en función de las propuestas divergentes. Como consecuencia, los tratamientos barajados para subsanarla varían considerablemente así como las concepciones antropológicas respecto al enfermo, pero también respecto al médico, su función y su papel en el tratamiento de la enfermedad. Al mismo tiempo, el debate se vuelve más delicado desde el momento en el que el estatuto de la disciplina psiquiátrica, -eminentemente científico o prioritariamente humanístico-,parece depender de la asunción ontológica que se adopte en torno a los fenómenos de salud y enfermedad. Siguiendo la corriente naturalista, cuyo máximo exponente desde la dé-

Thémata. Revista de Filosofía No60 (2019) pp.: 35-48. 
cada de 1970 es el filósofo Christopher Boorse, el estatuto ontológico de la enfermedad y la salud ha de basarse en criterios biológicos objetivos y libres de valores. La enfermedad se considera un defecto en el organismo al respecto de una norma biológica que conforma el diseño de la especie, y que puede medirse en términos estadísticos atendiendo al funcionamiento típico o habitual en los individuos sanos. Ahora bien, si esta cuestión ya es puesta en tela de juicio en el ámbito de la medicina somática por la corriente normativista, cuyo máximo exponente contemporáneo sería T. Engelhardt -para quien el estatuto ontológico de la enfermedad se basa en criterios sociales y culturales antes que biológicos-, en el terreno de la medicina psiquiátrica el debate adquiere otras dimensiones. Según ciertos psiquiatras y autores como Kraepelin (1856-1926) o Alf Ross (1899-1979), «no existe ninguna diferencia entre el diagnóstico de enfermedad mental y el de enfermedad somática» (Wulff, Pedersen y Rosenberg, 2002:152). De este modo, las enfermedades mentales tienen su fundamento objetivo en el ámbito de la fisiología, entendidas en tanto alteraciones ya sea del sistema nervioso central, ya sea debido a defectos genéticos que causarían un desequilibrio bioquímico en el organismo. En el extremo opuesto a esta concepción, el movimiento de la antipsiquiatría, cuyo máximo exponente es Thomas Szasz (1920-2012), defiende que la frontera entre el diagnóstico somático y el psiquiátrico es radical: «el concepto biológico de enfermedad mental constituye una ilusión peligrosa porque lleva a tratar problemas existenciales con fármacos [...]» (Wulff et al.,2002: 161). Así pues, lo que el paradigma de la psiquiatría biológica considera como "enfermedades», son, en su mayoría, problemas existenciales o éticos y su categorización en el sistema médico pasa por ser una convención. En esto parecen estar de acuerdo otros psiquiatras contemporáneos, ligados al paradigma de la psiquiatría existencial: «el sufrimiento humano con el que lidian los psiquiatras [...] es casi siempre de naturaleza psicológica, existencial y social, antes que biológica.» (Breggin,2003:41). Tal y como observan V. Aucouturier y S. Demazeux (2012:84), lo que Thomas Szasz pone en tela de juicio no es tanto el estatuto ontológico de la enfermedad sino «el uso social» que los psiquiatras hacen del término «enfermedad», crítica a la que no habrían respondido, en sus diversas propuestas, ni Christopher Boorse ni Tristram Engelhardt. De este modo, según Szasz, la disfunción de la psique pasa por ser nada más que una "metáfora», un "mito» trasladado al lenguaje médico, de una desviación frente a un código normativo de carácter ético, legal o existencial.

La concepción biologicista de la enfermedad lleva aparejada una perspectiva mecanicista del ser humano. En palabras del psiquiatra norteamericano Peter Breggin (2003: 44): «la adopción extendida del modelo mecánico [...] exige que nos pensemos a nosotros mismos como máquinas 
estropeadas o aparatos mecánicos defectuosos». Desde la perspectiva naturalista, el enfermo se pone en manos del médico, tal y como una máquina defectuosa es confiada a un técnico para su reparación. Esta visión despersonalizadora es criticada duramente por la psiquiatría existencial. Viktor Frankl, a lo largo de sus obras, denuncia esta deshumanización propia de la concepción naturalista en la que también se apoya el psicoanálisis, así como la atomización de la psique que esto conlleva (Frankl, 2012b: 33). Al cosificar al ser humano -al robotizarlo, concretamente- se asume que puede ser tratado parcialmente, como un compuesto de piezas separadas, y deja de considerárselo como algo global, integral y total. Esta crítica también la suscribe el normativismo contemporáneo, cuando propone que la enfermedad debe definirse tomando como base la totalidad que forma el ser humano y su entorno social (Kóvacs, 1998: 32) Pero no es a esta totalidad, entre el ser humano y su medio, a la que se refiere la psiquiatría existencial y, en particular, la logoterapia. Lo que la logoterapia añade a la dimensión biológica y psicológica del ser humano, no es la dimensión social que el paradigma biologicista habría ignorado, sino la dimensión noética, espiritual o noológica que caracteriza la constitución del fenómeno humano y lo diferencia del resto de animales y seres vivos, dimensión que el paradigma biologicista y normativista habrían simplemente evitado contemplar. Este reduccionismo se opera, pues, desde cualquier dominio parcial que pretenda haber agotado la totalidad del problema: «En cuanto la totalidad es proclamada, la biología se convierte en biologicismo, la psicología en psicologismo, y la sociología en sociologismo. En otras palabras, la ciencia se convierte en ideología.» (Frankl, 2012a: 30). Así, cualquier clase de reduccionismo es considerado por Viktor Frankl un "subhumanismo», además de una "perspectiva pseudocientífica» que subestima el fenómeno humano al reducir a un mero «epifenómeno», precisamente, lo que de humano hay en él (2012a: 28). La dimensión espiritual del ser humano permite integrar sus otras dos dimensiones, la psíquica y la somática, y en ella reside lo constitutivamente humano, esto es, la unidad trascendente de la conciencia y la responsabilidad (Frankl, 2008: 16) que permite concebir al ser humano como un ser dotado de libertad y de voluntad de sentido que se dirige siempre hacia algo trascendente y no meramente a la consecución de su supervivencia (Frankl, 2012a: 26 y Frankl, 2012b: 176). Así, Frankl se opone al "pandeterminismo» que, según él, caracteriza el enfoque antropológico de otras psicoterapias, pero no defiende, sin embargo, que el ser humano esté libre de cualquier condicionamiento (Frankl, 2012a: 26). Lo que la logoterapia enfatiza, partiendo de su posición antropológica, es la capacidad específicamente humana para la "autotrascendencia» y el «autodistanciamiento» con respecto a sus dimensiones somática y psicológica. Estas dos facultades son potenciadas por la logoterapia en la práctica 
clínica a través de dos prácticas específicas: la «derreflexión» (autodistanciamiento) y la «intención paradójica» (autotrascendencia). De este modo, dentro del ámbito de la psiquiatría existencial, la logoterapia, según Frankl, «ha sido reconocida [...] como la única escuela que ha tenido éxito en el desarrollo de lo que podría llamarse una técnica» (2012a: 15). Para tratar psicoterapéuticamente una clase de neurosis a la que Frankl denomina «noógena», la logoterapia se sirve de los recursos considerados exclusivamente humanos, como el humor y el distanciamiento consciente, así como la consciencia de su responsabilidad ante las elecciones a las que se enfrenta inevitablemente el ser humano a lo largo de una vida cuya plenitud dependerá del sentido («logos») que éste le otorgue. La ausencia de este sentido, el absurdo o vacío existencial, son estados constitutivos del fenómeno humano y no deben ser considerados como patológicos: «El vacío existencial no es una neurosis; o, de serlo, es una neurosis sociogénica o aun una yatrogénica, es decir, una neurosis ocasionada por el médico en su pretensión de curar» (Frankl, 2012a: 91). Ahora bien, si el vacío existencial no es una consecuencia ni un síntoma de la neurosis, sí puede terminar derivando en una. Una vez este vacío existencial propio de los seres humanos dotados con voluntad de sentido, ha afectado a la dimensión psicológica o a la dimensión somática, entonces sí estamos ante un caso de neurosis cuya etiología, no obstante, se inscribe en la dimensión noógena, y debe ser tratada por médicos especialistas en logoterapia (Frankl, 2012a: 93-94). Así pues, la logoterapia debe ser comprendida como el tratamiento principal para las neurosis noógenas, pero también como un complemento terapéutico para otros tipos de enfermedades, no solo mentales, y cuyo estatuto biológico, Frankl no pone en duda, como la esquizofrenia, las neurosis somáticas (cierto tipo de agorafobia), los procesos psicóticos, las parálisis nerviosas e incluso, las enfermedades somáticas incurables o terminales (Frankl, 2008). Porque, si el ser humano debe ser entendido como un todo global, la dimensión donde esta totalidad se hace efectiva y se integra debe ser prioritaria a la hora de definir en qué consiste la salud. De ahí que, a pesar del estatuto biológico de las enfermedades antes mencionadas, la psicoterapia todavía pueda ayudar a restaurar la salud incidiendo no en la dimensión biológica o psicológica sino en la dimensión noética. El enfoque de Frankl se aproxima, en ciertos puntos, al presentado por Robert M. Sade (1995: 520) cuando insta a distinguir entre "salud biológica» y «salud moral». Según Sade, podemos considerar que ciertas partes del cuerpo están sanas o enfermas, como hace el paradigma biologicista, pero "la idea de salud se aplica fundamentalmente a la totalidad de la persona» (1995: 523). Así pues, en el ámbito de la logoterapia, podríamos considerar que la "salud noética» no sólo es más importante sino también crucial para determinar e incidir sobre la enfermedad a nivel biológico, sea ésta somática o

Thémata. Revista de Filosofía No60 (2019) pp.: 35-48. 
psicológica. Por un lado, las alteraciones graves de la dimensión noética, tales como la pérdida total del sentido de la vida, pueden causar trastornos psicológicos y somáticos y, por otro, ante una enfermedad somática o psicológica, el fortalecimiento de la dimensión espiritual puede ayudar al enfermo a autodistanciarse de los procesos sufrientes y, lo que es más, a proporcionar un sentido («logos») a su sufrimiento ("pathos») (Frankl, 2008: 51-52). Ayudando al enfermo a dotar de sentido al sufrimiento, el logoterapeuta no busca eliminarlo, dado que éste a menudo es inevitable, sino hacer desaparecer la "desesperación» que, frecuentemente, lo acompaña (Frankl, 2012b: 176). Tanto Sade (1995: 521) como Kóvacs (1998: 38) consideran que la moral debe ser un elemento a tener en cuenta para una definición satisfactoria del término «salud». Frankl, ubicaría esa conciencia moral y esa responsabilidad en la dimensión «noética» o espiritual. Así como en el normativismo objetivista de Sade (1995: 522), los valores morales tienen un carácter universal y objetivo, y se prestan a ser «descubiertos" por los individuos, la logoterapia contempla estos valores como medios para llegar a "descubrir» el sentido: «Frankl en este sentido, dice que el camino hacia el sentido son los valores, y que el órgano mediante el cual se conoce o desvela el sentido es la conciencia moral» (Gengler, 2009: 202). El sentido que la voluntad humana es capaz de otorgar, consiste en un sentido personal que obedece al imperativo de un "suprasentido» objetivo y dado en el mundo, independiente de los seres humanos, que debe también ser descubierto por ellos. "Si ampliáramos nuestro horizonte, podríamos notar que disfrutamos de nuestra libertad, pero todavía no somos plenamente conscientes de nuestra responsabilidad. Si lo fuésemos nos daríamos cuenta de que el sentido está allí esperando que lo realicemos [...]» (Frankl, 2012a: 100). El suprasentido representa el sentido total del universo, el sentido de la totalidad misma, y cumple la función de un ideal al que el ser humano no puede responder (Frankl,2008: 55) pero hacia el que, sin embargo, siempre se orienta, pues: «el hombre vive por ideales y valores. La existencia humana no es auténtica, a menos que sea vivida en términos de autotrascendencia» (Frankl,2008: 58) Estos dos sentidos son calificados también como "sentido ontológico» (suprasentido) y "sentido existencial» (sentido personal). El primero implica un cuestionamiento ontológico sobre "por qué algo existe», mientras que el segundo consiste en una interrogación más modesta, dirigida al por qué de una existencia concreta que termina por constituir la respuesta del cómo y hacia qué meta dirigir la vida del existente (Gengler, 2009: 202). Esto es, la pregunta existencial por el sentido reconduce la voluntad de sentido de la que el ser humano está dotado y fortalece la dimensión crucial que lo constituye. $\mathrm{Ni}$ la supervivencia en términos biológicos ni tampoco la felicidad o el placer en términos morales, son metas que puedan proporcionarle al ser humano

Thémata. Revista de Filosofía No60 (2019) pp.: 35-48. 
el apoyo necesario para su salud noética. En palabras de Viktor Frankl: «Si el placer fuese realmente el sentido de la vida habría que llegar a la conclusión de que la vida carece, en rigor, de todo sentido» (2008: 64). ¿Qué sentido tendría entonces la vida -se preguntará Frankl- de una persona que está a las puertas de la muerte? La psicoterapia no tendría, efectivamente, ningún papel que cumplir en el caso de un condenado a muerte o de un enfermo terminal, dado que sus posibilidades de placer y supervivencia estarían, en ambos casos, amenazadas irreversiblemente. Los valores morales objetivos, ligados a la salud -dieta apropiada, ejercicio, etc-, que Sade (1995: 521) defiende como partes integrantes de la medicina, dejan de ser valiosos para una persona cuyas posibilidades de «florecer» son mínimas. Según la logoterapia, el bienestar no es, tal y como Sade postula, un fin en sí mismo. Las personas enfrentadas a situaciones terminales y trágicas tienen todavía la posibilidad de restaurar su salud o, al menos, de evitar perderla por completo, en el nivel más importante de su naturaleza, esto es, en la dimensión noética, y siempre a través de una dación de sentido; aplicando su capacidad para distanciarse y ejerciendo su libertad para otorgar sentido a lo que les sucede en cada momento. De esta forma, la salud no es un mero valor moral que forma parte del camino a una vida feliz o satisfactoria, sino que pasa a ser la condición que nos permite desarrollar plenamente las capacidades humanas, esto es, dotar de sentido tanto a la felicidad como a la infelicidad, ejercer la autorreflexión y la autotrascendencia en toda situación, especialmente en los casos donde nuestro sufrimiento es intenso. Cuando Kóvacs concibe la salud como «la habilidad para adaptarse a normas sociales razonables», especifica que se trata de una habilidad «mental y somática» (1998:36). Ahora bien, él mismo reconoce que el concepto de «norma social razonable» es impreciso, dado que depende de muchos factores $\mathrm{y}$, en último término, cuánto más tratemos de precisarlo, más adquirirá éste un carácter subjetivo y controvertido. Sin embargo, ofrece ejemplos de sociedades que no cumplen este requisito: la sociedad esclavista, la sociedad racista, y la sociedad nazi. Las personas que no logran adaptarse a estas sociedades no pueden ser consideradas enfermas dado que dichas sociedades los fuerza a vivir bajo normas no razonables (Kóvacs, 1998 :37-38). Ahora bien, es obvio que las personas obligadas a vivir en estas sociedades pueden, sin embargo, enfermar a causa de ellas. Si seguimos la línea marcada por Kóvacs, la salud sólo podría ser restaurada si el entorno social mismo fuese reconstituido, y la persona pudiese vivir bajo normas más razonables a las que poder adaptarse. No obstante, como superviviente de varios campos de concentración, Viktor Frankl no puede darle al entorno la importancia decisiva que Kóvacs le confiere. El pre-requisito para la salud de las personas no es la salud del entorno, tal y como Kóvacs afirma (1998:37), pues aún en el en- 
torno más degradante un ser humano puede conservar su salud, no gracias a una adaptación somática o psicológica, sino gracias a la integridad de su dimensión noética, de su «libertad interior». Analizando los cambios de carácter típicos de los reclusos del campo de concentración, Frankl defiende que más allá de sus fundamentos fisiológicos (hambre, insomnio, etc.) y psíquicos (complejo de inferioridad, etc.), éstos eran «en último término y sustancialmente, algo más: una actitud espiritual.»

El hombre conserva en todo caso y por difíciles que las condiciones sean, la libertad y la posibilidad de optar por o contra la influencia del medio en que vive.[...] Si nos preguntamos cuáles eran las razones que movían a estos hombres a dejarse arrastrar por las influencias somático-psíquicas del medio, a entregarse a aquellas sin luchar, tendremos que decir: se entregaban porque y cuando perdian su punto de apoyo espiritual.(Frankl, 2008:154)

En primer lugar, ni el bienestar, ni las circunstancias favorables del entorno son en sí mismos suficientes o necesarios para garantizar la salud noética del ser humano; en segundo lugar, sin la buena condición de esta dimensión humana, no puede hablarse propiamente de «salud». La enfermedad de la dimensión somática y psíquica, cuando no tiene su causa en la dimensión noética, necesita de la salud de esta última para poder ser enfrentada con efectividad. De ahí que la logoterapia tenga siempre su espacio de aplicación, ya sea como complemento de un tratamiento somático o psíquico, ya sea como tratamiento principal en caso de neurosis noógenas. La logoterapia debe ser practicada también con fines preventivos, en casos en los que el vacío existencial motivado por la falta de sentido, un rasgo natural y no patológico, propio de nuestra condición humana, amenaza con convertirse en una patología. La dimensión espiritual (noética, existencial; de carácter no empírico) debe ser, por tanto, prioritaria y fundamental a la hora de entrar a elaborar una definición satisfactoria de la salud.

\section{Aquí y Ahora: biopsiquiatría y farmacoterapia}

A pesar de que el enfoque biologicista de la psiquiatría es el que, tradicionalmente, suscribe el empleo recurrente a la farmacoterapia para tratar diversas clases de trastornos mentales, muchas escuelas de psiquiatría existencial también proponen la psicoterapia como complemento a un tratamiento farmacológico en el caso de patologías probadas, como la esquizofrenia o la psicosis. No obstante, el psiquiatra Peter Breggin, crítico con la industria farmacéutica, señala que «en nuestros días, incluso la psiquiatría humanista o existencial es propensa a recomendar medicación [...] cuando un paciente está especialmente estresado o no progresa a lo 
largo de la terapia» (2003:35). Aunque Viktor Frankl se opone el uso de medicamentos para tratar neurosis noógenas, sí considera legítimo el uso de medicamentos en patologías importantes, tales como «neurosis psicógenas». De este modo, cuando la etiología de una neurosis sea «muldimensional», el diagnóstico y la terapia también deberán serlo: «no existe una objeción a priori contra los shots and shocks. En los casos que en psiquiatría se denominan depresiones endógenas es perfectamente legítimo y justificado el uso de drogas y, en casos severos, aun aplicar tratamiento electro convulsivo» (Frankl,2012a:36-37). En estos casos, la logoterapia será empleada a modo de complemento, para incidir en la dimensión noética del paciente. No obstante, Breggin abre un debate importante cuando sugiere que la farmacoterapia merma, en ocasiones irreversiblemente, la capacidad de los seres humanos para hacer uso de sus facultades más elevadas, tales como ejercer la libertad, la responsabilidad o valorar desde una posición de distanciamiento (2003:43). De esta forma, cabe preguntarse cómo la logoterapia puede, a modo de complemento, ayudar a restaurar una dimensión espiritual, específicamente humana, que el propio tratamiento farmacológico al mismo tiempo deteriora y perjudica.

Sin llegar a considerar, como la antipsiquiatría, que la esquizofrenia podría tratarse mediante psicoterapia intensiva (Wulff et al., 2002: 152), Breggin está convencido de la incompatibilidad de ambas metodologías, la farmacoterapia y la psicoterapia existencial, que, además, enfrentan dos concepciones del ser humano opuestas e irreconciliables. El médico y filósofo francés, G. Canguilhem (1904-1995) consideraba todo movimiento «anti» como una moda en auge en al ámbito de la medicina -antipsiquiatría, antimedicalización, etc. (2004:92). No obstante, tal vez estos movimientos anti quieran venir a compensar un contramovimiento basado en el exceso indiscriminado: «Los pasados treinta años han sido testigos de un alarmante ciclo vicioso. La inflación diagnóstica ha conducido a un aumento explosivo en el uso de drogas psicotrópicas» (Frances,2013:77). El psiquiatra Frances Allen atribuye a la industria farmacéutica y al marketing de dicha industria un rol importante en dicha inflación desproporcionada que se refleja en la extensión del inventario de trastornos presentado por el DSM-5 (2013:84). Tal y como nos recuerda Breggin, a menudo, los pacientes desconocen la diferencia sustantiva entre los fármacos somáticos y los fármacos psiquiátricos: «Tomar drogas psiquiátricas no es como tomar insulina para diabetes. En psiquiatría, el "órgano principal" es el cerebro, y el cerebro es el asiento de nuestro pensamiento, de nuestro sentimiento mismo.»(2003:44). Esto es todavía más relevante en tanto existe la posibilidad de que estos fármacos se estén suministrando para trastornos que pueden tener su causa en la dimensión noética, como apuntaba Viktor Frankl, y deberían estar siendo tratados con psicoterapia. No obstante, 
y en el peor de los casos, pudiera ser que estos tratamientos agresivos se suministrasen para conductas y estados «normales», como muchos de los que se incluyen en el DSM-5, «enfermedades» a las que Frances denomina "las modas» del futuro: «Todas poseen síntomas que son parte de la vida diaria y comúnmente presentes en la población general. [...] ninguna tiene un tratamiento probado que sea efectivo. Todas conducen probablemente a un tratamiento innecesario, en ocasiones nocivo.»(2013:176). Lo más grave de posicionarse ante el debate sobre el estatuto ontológico de la enfermedad, y de asentar bien los criterios para su adecuada definición, no es simplemente que dicha posición podría implicar la posibilidad de estigmatizar de forma injustificada a las personas como «enfermos», sino sobre todo la elección del tratamiento que se sigue de dicho compromiso ontológico. Tal y como declara Breggin (2003:45), la biopsiquiatría pretende basarse simplemente en datos biológicos y experimentos empíricos, sin atender a asunciones de carácter ontológico sobre la naturaleza del ser humano o los valores. No obstante, todo médico asume, ya sea reflexiva o pre-reflexivamente una toma de partido ontológica y antropológica que determina la elección del tratamiento a seguir. Como cualquier ser humano, el médico comprende e interpreta la realidad antes de actuar. Esto vale también para el paciente-consumidor: «la mayor parte de las personas aceptan el concepto naturalista del hombre siendo una consecuencia [...] que los ansiolíticos y los antidepresivos se han convertido en un artículo comercial esencial en todo el mundo». (Wulff et al., 2002:177). Georges Canguilhem, por ejemplo, reivindicaba la necesidad de un punto intermedio entre dos clases importantes de reduccionismo, a saber: la concepción exclusivamente biologicista de la salud, con su consecuente puesta en práctica a través del modelo médico predominante, por un lado, y el recurso a un planteamiento psicologista, abstracto y difuso, carente de la debida formación científica.

Si algunos médicos [...] descuidan -menos tal vez por principio que por falta de tiempo- preguntarse pacientemente por el eventual desamparo afectivo de sus pacientes, ¿autoriza esto a considerarlos inferiores respecto del primer terapeuta que aparezca reivindicando la psicosomática? ¿Se hallará éste más calificado para lograr la curación de una obesidad, fruto primero de comportamientos alimentarios de compensación afectiva, pero determinada ahora por un desarreglo tioideo o suprarrenal? A la hora de hacerse reduccionista en terapétuica, ¿vale más el psicologismo que el fisiologismo? (Canguilhem, 2004:94-95)

Como defiende John Saunders (2000), el ejercicio de la medicina debe ser considerado tanto un arte como una ciencia. Y la perspectiva ideal es que sea aprendido y aplicado como tal, precisamente con el fin de evitar caer en prácticas y aproximaciones reduccionistas. No obstante, la parte "artística" de la medicina es la más difícil de enseñar, dado que no con- 
siste en ninguna teoría que pueda ser aprendida de memoria. Se apoya, más bien, en la experiencia humanista del médico a través del curso de su profesión, y requiere de estrategias relacionales ligadas a una perspectiva de segunda persona. Esta perspectiva, según Michael Pauen, no es totalmente subjetiva ni tampoco objetiva, sino intersubjetiva, dado que "se emplea la propia experiencia para entender las creencias, los deseos y las emociones de los otros» (2012:38). Sin embargo, el propio modelo médico predominante es reacio a la terapia "basada en la conversación", es decir, a la psicoterapia como método de tratamiento médico, la cual está más orientada a la relación médico-paciente en términos de indagación afectiva y aproximación humanista. De hecho, ciertas teorías de la mente radicalmente fisicalistas, partidarias de la identificación completa entre estados mentales y estados cerebrales, han influido decisivamente en la concepción de la psiquiatría dentro del modelo biomédico. La consecuencia de esto es que la psicoterapia a menudo es considerada «un tratamiento pre-científico, esencialmente mágico: los psicoterapeutas son, como si dijésemos, una especie de doctores brujos» (Matthews, 2007:10). Por otro lado, es indudable que el enfoque psicoterapéutico exige respetar ciertos procesos relativos al tiempo, como bien advertía Canguilhem, que las dinámicas del modelo médico actual no favorecen; a su vez, este modelo no deja de ser el resultado de una dinámica social de aceleración e inmediatez en el que están inmersos tanto médicos como pacientes a la hora de entender el proceso de enfermedad y de recuperación. El modelo biomédico, hoy asumido casi globalmente, ¿no forma parte de esa "política de la velocidad" que tanto denunció el filósofo Paul Virilio? Este pensador francés hablaba de una "ritmología de la vida pública" (1997:66), una suerte de tempo social, que no deja de acelerarse, y que, en nuestra opinión, no podemos obviar a la hora de tratar el modelo médico dominante y también el recurso, abusivo según ciertos médicos, a la farmacología.

En este sentido, para poner el foco sobre la dimensión noética de la salud, sería necesario replantearse todo un escenario de valores, criterios y prioridades, a la hora de considerarnos a nosotros mismos como seres humanos finitos, en constante cambio y movimiento. La trascendencia como motor de vida, tal y como Frankl la expone, así como la dotación de un sentido a todo lo que nos ocurre, conlleva un ejercicio de reflexión que desafía un modo concreto de temporalizar la vida, y de temporalizarnos a nosotros mismos, forjado a base de coleccionar instantes efímeros, fácilmente olvidables y dispuestos a ser automáticamente reemplazados por los siguientes. La dicotomía salud/enfermedad se arriesga a ser subsumida en esta modalidad consumista de la experiencia, bajo cuya inercia la clasificación de ambos estados se asume de forma mecánica y mecanizable, olvidándose 
con ello el componente precisamente noético (y trascendental) que subyace a la pluralidad de dimensiones de las que se compone cada ser humano.

\section{Conclusiones}

En su intento por rehumanizar la psiquiatría y la psicoterapia, lo que la logoterapia cuestiona no es el sustrato fisiológico de las enfermedades somáticas o psicológicas, sino toda definición de «salud» que sólo contemple: a) alguna de estas dos dimensiones, b) ambas, c) cualquiera de ellas o ambas y la dimensión social. Cualquier tipo de consideración de la salud que excluya la dimensión noética supone un reduccionismo, en el que Frankl veía «la máscara del nihilismo» (2012a:30). El existenzanalyse rechaza entender la salud como un valor imprescindible de un camino vital cuya meta es, simplemente, el bienestar o la felicidad. Todas estas propuestas, ya sean biologicistas o normativistas, objetivistas o subjetivistas, se olvidan de la única dimensión que hace "humano» al ser humano. Al subestimar sus capacidades específicas, -su voluntad de sentido, su libertad y su responsabilidad como atributos de una conciencia vinculada a la trascendencia-, resulta sencillo clasificar como «enfermedades» psíquicas lo que tan solo son rasgos intrínsecos de la propia condición humana: el vacío existencial, la angustia, la ansiedad, etc. Cuando estos estados se vuelven graves reflejan toda una sintomatología en la dimensión psíquica: se trata de las «neurosis noogénicas» que deben ser tratadas principalmente con logoterapia u otras psicoterapias humanísticas y, de ningún modo, con farmacoterapia. En última instancia, y aunque un medio social adverso pueda haber contribuido a la generación de dicha neurosis, la logoterapia devuelve al ser humano la plena responsabilidad que le corresponde, en tanto ser dotado de una libertad interna que desafía tanto el medio social como las circunstancias psíquicas y somáticas en las que la persona se ve implicada. No obstante, en casos donde la etiología es múltiple, el tratamiento también ha de serlo. "La logoterapia no es una panacea", advierte, en numerosas ocasiones, Viktor Frankl, y por lo tanto puede ser combinada con diversas psicoterapias, con la religión misma y con la farmacoterapia. Así pues, la logoterapia propone que el estatuto ontológico de muchas de las enfermedades de las que se ocupa la psiquiatría no es exclusivamente biológico o social, sino, ante todo, noológico, y debe ser abordado efectivamente como tal. 


\section{Bibliografía}

Aucouturier,V.; Demazeux, S.: "The concept of mental disorder" en R. Cooper; H. Carel (Ed), Health, Illness and disease: philosophical essais. New York: Acumen publisher, 2012, pp. 75-89.

Breggin, P.: "Psychopharmacology and human values" en Journal of Humanistic Psychology, 43 (34), 2003, pp. 34-49. Doi: 10.1177/0022167802250729

Brennan, J.: Historia y sistemas de la psicología. México: Practice hall, 1999.

Tobías, C.; García-Valdecasas, J.: "Psicoterapias humanístico-existenciales: fundamentos filosóficos y metodológicos" en Revista Asociación Española de Neuropsiquiatría, XXIX (104), 2009, pp. 437-453.

Canguilhem, G.: Escritos sobre la medicina. Buenos Aires: Amorrortu, 2004.

Frances, A.: Saving normal. New York: Morrow, 2013.

Frankl, V.: Fundamentos y aplicaciones de la logoterapia. Barcelona: Herder, 2012a.

Frankl, V.: El hombre en busca del sentido último. Barcelona. Paidós, 2012b.

Frankl, V.: Psicoanálisis y existencialismo. De la psicoterapia a la logoterapia. México: Fondo de cultura ecónomica, 2008.

Gengler, J.: "Análisis existencial y logoterapia: bases teóricas para la práctica clínica". Psiquiatría y salud mental, XXVI (3), 2009, pp.200-209.

Kovács, J.: "The concept of health and disease" en Medicine, Health Care and Philosophy 1, 1998, pp. 31-39.

Matthews, E.: Body-subjects and disordered minds. Oxford: Oxford University Press, 2007.

Pauen, M: "The Second-person perspective" en Inquiry: an interdisplicinary Journal of Philosophy 55 (1), 2012, pp. 33-49. Doi: 10.1080/0020174X.2012.643623

Sade, R.: "A theory of health and disease: the objectivist-subjectivist dichotomy" en The journal of Medicine and Philosophy, 20, 1995, pp. 513-525.

Saunders, J.: "The practice of clinical medicine as an art and as a science" en Journal of Medical Ethics: Medical Humanities, 26, 2000, pp. 18-22.

Watters, E.:Crazy like us: the globalization of the american psyche. New York: Free Press, 1995.

Virilio, P.: El Cibermundo, la política de lo peor. Madrid: Cátedra, 1997.

Wulff, H.; Pedersen, S.; Rosenberg, R.: Introducción a la filosofía de la medicina. Madrid: Triacastela, 2002. 